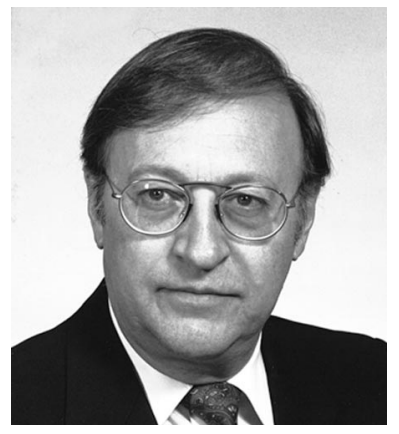

\section{Y2K is finally here! What will be our next fear, our future?}

Year 2000 has finally arrived. The year that was anxiously awaited by numerous people to see if something abnormal would happen. For the first editorial of this year, the temptation was very strong to do a satiric review of all the predictions for large disasters. If we look back in history, every turn of the century makes human beings anxious and causes them to prepare themselves for the worst. We may understand this kind of problem in earlier times when people did not have access to information or were living in a world in which scientific evidence of cataclysm was not documented. Is it curious that although we now live in a world where information is predominant, we still have seen all kinds of predictions, most no more realistic than another.

The big cataclysm that is supposed to block all our computers demonstrates that we are not able to build machines comparable to human beings. By the time this editorial is published, we will all know if the big bug was in fact a very powerful tool to make a profit, or if we were really in such a bad situation. This bug is so marvelous that engineers and scientists have even tested electrical appliances containing very low technology chips. Those tests were done to convince everyone that nothing would happen. What will happen to our optical equipment since we know that optical instruments are made of more than $70 \%$ electronics?

The optics community also encountered our own bug. We spent time and effort to define optics as a science, and it seems that fear did overcome the desire of a part of our community to convince our granting bodies that we work in a field called optical science and engineering. It seems that our community is well balanced, showing an almost exact split between basic scientists and engineers. From my point of view, the bug came from the fact that most of the people who believed in the unification did not talk about this unified face; rather, the debate was mainly oriented on an economic point of view. This was very well analyzed by my predecessor. I bring up the topic again because it reminds me of the time I decided to go into graduate studies in optics. It was a period when most of my colleagues were choosing nuclear physics, or what we today call subatomic physics. We were all physicists or physics engineers, but often we were not considered part of this physics community. Optical physics was not serious enough to be considered. I was feeling the same with the kind of debate we had this time inside the so-called optics community. Time will show us again what was the reality that we did not foresee.

Once the bug is behind us, what is the future of optics as a discipline? Numerous initiatives have been started to show that optics is important for everyone in our everyday life. We hopefully will not see any new fears, and our future appears to be sunny.

SPIE and OSA worked together to develop a K-12 kit for showing youths that light is a part of their life. They have used light from their first day to discover the world around them, and they must learn that light will also be a very important part of their future. Optical scientists and engineers must start teaching what they really think that optics is, and hopefully we will see many more youths joining sciences, especially in our field.

Another aspect of the future is also perceptible if we consider the increasing number of optics-related industries. Their visibility is increased by clustering, a phenomenon we can observe in many regions. This kind of "coopetition' (cooperation in competition) is perceived by many as the normal way of developing new markets. Numerous governments have started such groups in various fields, and it seems that in optics we are doing a better job of clustering.

Our future will shine if our youths enter science, especially in optics; then the optics industry will continue to develop. We need to design college and university programs that will make excellent scientists who will discover new applications through their devotion to optical science and engineering. Moreover, numerous governments want to favor the establishment of optics industries in their countries. My government wants to help the development of such industries by declaring Québec City an 'Optics City,', and special support will be given to start and develop new industries there. Similar things are being 
done in many other areas where optics clusters have been started, such as Tucson, Orlando, Singapore, etc. I would like to be able to review all Optics Cities and Photonics Cities that have been started and put forward in the world, but it would take up all this year's editorials.

It is obvious that optics is developing very rapidly and is entering all aspects of our lives.

The real turning of the century will now take place without fear, allowing everyone to see how optics is speeding communication and allowing development of new test, measurement, and analysis devices that will give access to things that most of the population did not care about previously.
However, one important thing that will come out of the marriage of optics and electronics is electronic publication. Optical Engineering will take advantage of that by encouraging manuscript submissions through the Internet. This is what I want to encourage during my one-year term as $\mathrm{OE}$ Editor. We are looking forward to developing other means to increase our publication strength, but this will be part of another story.

Have a Happy and Prosperous Year 2000.

Roger A. Lessard Editor

\section{Getting the Word Out: Electronic Submissions to Optical Engineering}

Since the introduction of electronic submission of manuscripts to Optical Engineering, we have found that this process has helped to reduce the time required to review a manuscript. This is because e-submissions are easily transmitted via e-mail to the editorial office and to the editor and editorial board members, as well as to reviewers who wish to receive manuscripts electronically. This method helps to eliminate delays associated with sending paper copies of the manuscript through the mail. In addition, submissions received electronically can be processed efficiently. Authors may send the file as an e-mail attachment or via ftp to the Managing Editor. E-submissions are accepted in either PDF or PostScript format. Complete instructions for submitting a paper can be found on the Optical Engineering web page http://www.spie.org/web/journals/oe_infocontrib.html and the Information for Contributors page at the back of this issue. 